\title{
Remoção parcial do tecido cariado em dentes permanentes: uma revisão integrativa da literatura
}

\author{
Partial caries removal in permanent teeth: an integrative review
}

\begin{abstract}
Joyce Figueira de Araújo, Érica Martins Valois, ${ }^{1}$ Andréa Dias Neves Lago, ${ }^{2}$ Breno Mont Alverne Haddad da Silva, ${ }^{2}$ José Ferreira Costa, ${ }^{2}$ Leily Macedo Firoozmand ${ }^{2}$ 'Pós-Graduação em Odontologia, Faculdade de Odontologia, Universidade Federal do Maranhão, São Luís, MA, Brasil

${ }^{2}$ Departamento de Dentística, Faculdade de Odontologia, Universidade Federal do Maranhão, São Luís, MA, Brasil

- Os autores declaram que não há conflito de interesse.

\section{Resumo}

Objetivo: realizar uma revisão integrativa da literatura a respeito da RPTC em dentes permanentes considerando técnicas, materiais comumente aplicados e taxas de sucesso. Material e Métodos: : foi realizada uma busca nas bases de dados Pubmed e Scielo nos últimos 20 anos (de 1996 a 2016), seguindo critérios de inclusão e exclusão pré-determinados. Resultados: foram identificados dez artigos com texto completo que se enquadravam nos critérios de inclusão e exclusão. Quanto aos tipos de tratamentos empregados na RPTC, se destacaram as técnicas de remoção em sessão única e duas sessões. Os materiais mais utilizados, isoladamente ou associados, foram cimento de hidróxido de cálcio, óxido de zinco e eugenol, e cimento ionômero de vidro. As técnicas de RPTC apresentaram índices de sucesso entre $53 \%$ e $99 \%$, melhores que a técnica da RTTC que ficou entre 37,5\% e 75\%.Conclusão: a RPTC parece ser uma alternativa viável para o tratamento de cáries profundas em dentes permanentes, principalmente quando realizada em sessão única.
\end{abstract}

Palavras-chave: Cárie dentária; Dentes permanentes; Proteção pulpar indireta; Terapia expectante.

\section{ABSTRACT}

Objective: to conduct an integrative review on the removal of partial caries (RPC) in permanent teeth based on analyzing the various techniques performed, routinely applied materials, and success rates. Material and Methods: PubMed and Medline databases were searched for the period between 1996 and 2016 , and 10 full text articles that followed the previously determined inclusion and exclusion criteria were selected. Results: RPC was most frequently performed in one session or in two sessions. The materials were routinely used either alone or in combination with calcium hydroxide cement, zinc oxide-eugenol cement, and glass ionomer cement. The success rates of the RPC techniques ranged from $53 \%$ to $99 \%$, and the success rates of the total removal of caries ranged from $37.5 \%$ to $75 \%$. Conclusion: RPC, particularly in a single session, may be used as an alternative to treat deep carious lesions in permanent teeth.

Keywords: Carious lesion; Permanent teeth; Indirect pulpar therapy; Watchful waiting.

\section{Introdução}

$\mathbf{N}$ os últimos anos, ensaios clínicos vêm demonstrando os benefícios da remoção parcial do tecido cariado (RPTC) em cavidades profundas de dentes permanentes. ${ }^{1-4}$ No entanto, o tratamento dessas lesões ainda é uma questão bastante discutida, especialmente em relação às técnicas, materiais e índices de sucesso encontrados na literatura.

Por muito tempo o tratamento preconizado para a cárie dental consistiu na remoção de toda a dentina infectada e desmineralizada, mais conhecida como remoção total do tecido cariado (RTTC), com o objetivo de evitar futuras atividades cariogênicas e fornecer uma base bem mineralizada da dentina para realização da restauração. ${ }^{5}$ Contudo, a grande desvantagem desta técnica é o risco de exposição pulpar durante o tratamento em cavidades profundas. ${ }^{3}$

Devido ao risco elevado de exposição pulpar durante a RTTC ${ }^{3}$, houve a busca por tratamentos mais conservadores, sendo atualmente discutida a RPTC, com intuito de diminuir e prevenir a exposição pulpar, ${ }^{6-9}$ suportados pelo conhecimento de que a RTTC não é necessariamente um indicador para o sucesso do tratamento das lesões cariosas. ${ }^{4}$

Com o advento da Odontologia conservadora, propõe-se a preservação de uma camada da dentina desmineralizada sobre a polpa e a aplicação de um material forrador para induzir a formação de dentina terciária, selando a cavidade temporariamente, antes de realizar a restauração definitiva no dente. ${ }^{7} \mathrm{O}$ selamento da cavidade com materiais restauradores sobre a dentina cariada é capaz de estacionar a progressão da cárie por pelo menos 10 anos, indicando a não necessidade da remoção completa para a obtenção de sucesso clínico. ${ }^{1}$ Além disso, o correto selamento da dentina cariada diminui significativamente a microbiota sobrevivente, ${ }^{9}$ aumenta a dureza e reorganização da dentina, independente do material forrador utilizado. ${ }^{7}$

Duas linhas têm sido bastante investigadas: a RPTC em dois passos ou stepwise excavation e a RPTC em sessão única, considerada conservadora. ${ }^{5}$ A RPTC em dois passos consiste na remoção de parte da dentina cariada, deixando apenas aquela localizada na parede de fundo, seguida da realização do selamento provisório. Em uma segunda sessão, a cavidade é reaberta e realiza-se a remoção completa do tecido cariado, para posterior restauração definitiva. ${ }^{3}$ Já a RPTC em sessão única consiste na manutenção da dentina desmineralizada da região mais próxima à polpa e realização do selamento definitivo com um material biocompatível, inviabilizando o crescimento de micro-organismos capazes de dar continuidade ao avanço da doença. ${ }^{1}$

Ambas as técnicas reduzem significativamente o risco de ocorrer exposição pulpar e podem diminuir as complicações pós-operatórias advindas da remoção total do tecido cariado, ${ }^{10}$ entretanto ainda discutem-se as chances de sucesso/insucesso quando comparadas à remoção total. Além disso, escassos estudos clínicos 
longitudinais demonstram a efetividade das técnicas RPTC de uma ou duas sessões em dentes permanentes. Dessa forma, o objetivo deste trabalho foi realizar uma revisão integrativa da literatura a fim de se obter uma análise embasada em resultados clínicos, que pudessem orientar o cirurgião-dentista clínico a respeito das estratégias de RPTC em dentes permanentes tais como: técnicas, materiais comumente aplicados e as taxas de sucesso de cada uma delas, de modo a dar maior segurança em sua aplicabilidade.

\section{Material e Métodos}

Esta revisão integrativa foi realizada por meio de uma busca nas bases de dados Pubmed e Scielo nos últimos 20 anos (de 1996 a 2016), contendo as seguintes palavras-chave isoladas ou combinadas: "caries removal partial" e "excavation dentin" ou "deep lesions" ou "deep caries"; "stepwise excavation" e "carious lesion sealed", em inglês e português.

Os critérios de inclusão pré-determinados para o artigo foram: estudos clínicos randomizados ou não, abordando o tema RPTC, entre os anos de 1996 e 2016, publicados nos idiomas português ou inglês.

Após a seleção inicial, foram excluídos todos os artigos repetidos; aqueles que não contemplaram pelo menos duas palavras- chave no título e/ou resumo; aqueles que abordaram o tema RPTC exclusivamente em dentes decíduos e os estudos clínicos que não descreveram nos resultados as taxas de sucesso e insucesso das técnicas. Por fim, os artigos pré-selecionados foram lidos na íntegra por dois autores devidamente calibrados para determinar a amostra final e construir tabelas com as informações mais relevantes dos artigos selecionados.

\section{Resultados}

Foram identificados 157 artigos com texto completo nas bases de dados pesquisadas. Após a análise dos critérios de inclusão e exclusão pré-estabelecidos e leitura dos artigos na íntegra, foram selecionados 10 artigos científicos que abordavam a questão investigada nessa revisão. Dentre as informações obtidas dos estudos clínicos selecionados, destacou-se:

a) população, tipos de dentes tratados e materiais de escolha envolvidos em cada técnica (Tabela 1); b) tipos de tratamentos empregados para remoção da dentina cariada em cavidades profundas de dentes permanentes, bem como as taxas de sucesso e causas de insucesso (Tabela 2); c) tempo de acompanhamento, o número total e tipo de avaliações (Tabela 3).

Tabela 1. População, dentes tratados e materiais restauradores utilizados nos artigos avaliados

\begin{tabular}{|c|c|c|c|}
\hline Autor & População & Dentes tratados & Materiais restauradores \\
\hline $\begin{array}{l}\text { Leksell et } \\
\text { al., } 1996\end{array}$ & $\begin{array}{l}116 \text { pacientes } \\
6-16 \text { anos }\end{array}$ & $\begin{array}{l}127 \text { dentes permanentes } \\
\text { posteriores }\end{array}$ & $\begin{array}{l}\text { RPTC } 2 \text { sessões: } 1 \text { a visita - } \mathrm{CaOH}+\text { óxido de zinco e eugenol(ZOE) +res- } \\
\text { tauração. } 2 \text { a visita - após 8-24 semanas, } \mathrm{CaOH}+\mathrm{ZOE}+\text { restauração }\end{array}$ \\
\hline $\begin{array}{l}\text { Oliveira et } \\
\text { al., } 2006\end{array}$ & $\begin{array}{l}27 \text { pacientes } \\
12-23 \text { anos }\end{array}$ & $\begin{array}{l}32 \text { dentes permanentes } \\
\text { posteriores jovens }\end{array}$ & $\begin{array}{l}\text { RPTC } 2 \text { sessões: Hidróxido de Cálcio }(\mathrm{CaOH})+\text { Restauração provisória } \\
\text { (IRM). Após } 6 \text { a } 7 \text { meses, restauração definitiva }(\mathrm{CaOH}+\text { resina com- } \\
\text { posta) }\end{array}$ \\
\hline $\begin{array}{l}\text { Maltz et al., } \\
2007\end{array}$ & $\begin{array}{l}27 \text { pacientes } \\
12-23 \text { anos }\end{array}$ & $\begin{array}{l}32 \text { dentes permanentes } \\
\text { posteriores jovens }\end{array}$ & $\begin{array}{l}\text { RPTC } 2 \text { sessões: } \mathrm{CaOH}+\mathrm{IRM} \text {. Após } 6 \text { a } 7 \text { meses, restauração definitiva } \\
\text { (CaOH+resina composta) }\end{array}$ \\
\hline $\begin{array}{l}\text { Orhan et al., } \\
2010\end{array}$ & $\begin{array}{l}123 \text { pacientes } \\
4-15 \text { anos }\end{array}$ & $\begin{array}{l}60 \text { dentes permanentes } \\
\text { posteriores jovens }\end{array}$ & $\begin{array}{l}\text { RPTC } 1 \text { sessão: } \mathrm{CaOH}+\text { restauração (CIV+resina) } \\
\text { RPTC } 2 \text { sessões: } \mathrm{CaOH}+\mathrm{IRM}(1 \mathrm{a}) ; \mathrm{CaOH}+\mathrm{CIV}+\text { resina }(2 \mathrm{a}) .\end{array}$ \\
\hline $\begin{array}{l}\text { Gruythuysen } \\
\text { et al. } 2010\end{array}$ & $\begin{array}{l}66 \text { pacientes } \\
4-18 \text { anos }\end{array}$ & $\begin{array}{l}45 \text { dentes permanentes } \\
\text { posteriores jovens }\end{array}$ & RPTC 1 sessão: Forramento com CIV modificado por resina + resina \\
\hline $\begin{array}{l}\text { Bjørndal et } \\
\text { al. } 2010\end{array}$ & $\begin{array}{l}314 \text { pacientes } \\
25-38 \text { anos }\end{array}$ & $\begin{array}{l}314 \text { dentes permanentes } \\
\text { posteriores }\end{array}$ & $\begin{array}{l}\text { RPTC } 2 \text { sessões: } \\
1^{\text {a }} \text { visita }-\mathrm{CaOH}+\mathrm{CIV} \\
\text { 2a visita }-8-12 \text { semanasdepois, } \mathrm{CaOH}+\text { resina } \\
\text { RTTC: após } 8-12 \text { semanas, CIV substituído por resina }\end{array}$ \\
\hline $\begin{array}{l}\text { Maltz et al., } \\
2012\end{array}$ & $\begin{array}{l}233 \text { pacientes } \\
6-53 \text { anos }\end{array}$ & $\begin{array}{l}299 \text { dentes permanentes } \\
\text { posteriores }\end{array}$ & $\begin{array}{l}\text { RPTC } 1 \text { sessão: } \mathrm{CIV}+\text { resina } \\
\text { RPTC } 2 \text { sessões: } \\
1^{\text {a }} \text { visita - CaOH+IRM. } \\
\text { 2a visita - após } 90 \text { dias- }\end{array}$ \\
\hline $\begin{array}{l}\text { Maltz et al., } \\
2013\end{array}$ & $\begin{array}{l}233 \text { pacientes } \\
6-53 \text { anos }\end{array}$ & $\begin{array}{l}299 \text { dentes permanentes } \\
\text { posteriores }\end{array}$ & $\begin{array}{l}\text { RPTC } 1 \text { sessão: CIV+resina } \\
\text { RPTC } 2 \text { sessões: } \\
\text { 1a visita - CaOH+IRM. } \\
\text { 2a visita - após } 90 \text { dias-remoção total + resina }\end{array}$ \\
\hline $\begin{array}{l}\text { Petrou et } \\
\text { al., } 2014\end{array}$ & $\begin{array}{l}86 \text { pacientes } \\
\text { Média da idade } \\
-17,2\end{array}$ & $\begin{array}{l}86 \text { dentes permanentes } \\
\text { posteriores }\end{array}$ & $\begin{array}{l}\text { RPTC } 2 \text { sessões: } 1 \text { a visita - CaOH ou MTA ou Portland cement + CIV ou } \\
\text { IRM } \\
\text { 2a visita - após } 6 \text { meses, remoção tecido afetado+restauração defini- } \\
\text { tiva }\end{array}$ \\
\hline $\begin{array}{l}\text { Jardim et } \\
\text { al., } 2015\end{array}$ & 238 pacientes & 127 dentes permanentes & $\begin{array}{l}\text { RPTC } 2 \text { sessões: } \mathrm{CaOH}+\text { IRM. Após } 90 \text { dias, restauração definitiva } \\
\text { (CIV+amálgama ou resina) } \\
\text { RPTC } 1 \text { sessão: CIV+amálgama ou resina }\end{array}$ \\
\hline
\end{tabular}


Tabela 2. Índice de sucesso de acordo com o tipo de tratamento empregado

\begin{tabular}{|c|c|c|c|c|}
\hline Autor & Tipo de tratamento & Taxas de sucesso & Causas do insucesso & Perdas de seguimento \\
\hline $\begin{array}{l}\text { Leksell et al., } \\
1996\end{array}$ & $\begin{array}{l}\text { RPTC } 2 \text { sessões } \\
\text { RTTC }\end{array}$ & $\begin{array}{l}\text { 82,5\% (RPTC } 2 \text { sessões) } \\
37,5 \% \text { (RTTC) } \\
\end{array}$ & Exposição pulpar & - \\
\hline $\begin{array}{l}\text { Oliveira et al., } \\
2006\end{array}$ & RPTC 2 sessões & $93,57 \%$ & Necrose e exposição pulpar & $6 \%$ (6-7 meses) \\
\hline $\begin{array}{l}\text { Maltz et al., } \\
2007\end{array}$ & RPTC 2 sessões & $\begin{array}{l}93,57 \% \text { ( } 6-7 \text { meses }) \\
93,57 \% \text { (14-18 meses) } \\
90 \% \text { (36-45 meses) } \\
\end{array}$ & $\begin{array}{l}\text { Necrose, exposição pulpar, } \\
\text { fratura e substituiçãao de res- } \\
\text { tauração }\end{array}$ & \begin{tabular}{|l}
$-(6-7$ meses $)$ \\
$25 \%$ (14-18 meses) \\
$25 \%$ (36-45 meses) \\
\end{tabular} \\
\hline $\begin{array}{l}\text { Bjørndal et al., } \\
2010\end{array}$ & RPTC 2 sessões e RTTC & $\begin{array}{l}74,1 \% \text { (RPTC } 2 \text { sessões) } \\
62,4 \% \text { (escavação total) } \\
\end{array}$ & $\begin{array}{l}\text { Dor, radioluscência apical, } \\
\text { necrose e exposição pulpar }\end{array}$ & - \\
\hline $\begin{array}{l}\text { Gruythuysen } \\
\text { et al., } 2010\end{array}$ & RPTC 1 sessão & $93 \%$ & Reabsorção apical & - \\
\hline $\begin{array}{l}\text { Orhan et al., } \\
2010\end{array}$ & $\begin{array}{l}\text { RPTC } 1 \text { sessão, } 2 \text { sessões } \\
\text { e RTTC }\end{array}$ & $\begin{array}{l}\text { 95\% (RPTC } 1 \text { sessão) } \\
94 \% \text { (RPTC } 2 \text { sessões) } \\
75 \% \text { (escavação total) } \\
\end{array}$ & Exposição pulpar & - \\
\hline $\begin{array}{l}\text { Maltz et al., } \\
2012\end{array}$ & $\begin{array}{l}\text { RPTC } 1 \text { sessão e RPTC } 2 \\
\text { sessões }\end{array}$ & $\begin{array}{l}\text { 91\% (RPTC } 1 \text { sessão) } \\
69 \% \text { (RPTC } 2 \text { sessões }\end{array}$ & $\begin{array}{l}\text { Pulpite, necrose, exposição } \\
\text { pulpar, osteíte, exodontia e } \\
\text { fratura }\end{array}$ & $\begin{array}{l}\text { 26,3\% (RPTC } 1 \text { sessão) } \\
31 \% \text { (RPTC } 2 \text { sessões) }\end{array}$ \\
\hline $\begin{array}{l}\text { Maltz et al., } \\
2013\end{array}$ & $\begin{array}{l}\text { RPTC } 1 \text { sessão e RPTC } 2 \\
\text { sessões }\end{array}$ & $\begin{array}{l}\text { 99\% (RPTC } 1 \text { sessão) } \\
86 \% \text { (RPTC } 2 \text { sessões) } \\
\end{array}$ & $\begin{array}{l}\text { Pulpite, osteíte, necrose, trata- } \\
\text { mento endodôntico }\end{array}$ & $\begin{array}{l}26 \% \text { (RPTC } 1 \text { sessão) } \\
31 \% \text { (RPTC } 2 \text { sessões) }\end{array}$ \\
\hline $\begin{array}{l}\text { Petrou et al., } \\
2014\end{array}$ & RPTC 2 sessões & $90,3 \%$ & Tratamento endodôntico & $27,9 \%$ \\
\hline $\begin{array}{l}\text { Jardim et al., } \\
2015\end{array}$ & $\begin{array}{l}\text { RPTC } 1 \text { sessão e RPTC } 2 \\
\text { sessões }\end{array}$ & $\begin{array}{l}\text { RPTC } 1 \text { sessão: } \\
99 \% \text { (18 meses) } \\
91 \% \text { (36 meses) } \\
78 \% \text { (60 meses) } \\
60 \% \text { (72 meses) } \\
\text { RPTC } 2 \text { sessões: } \\
86 \% \text { (18 meses) } \\
53 \% \text { (60 meses) } \\
32 \% \text { (72 meses) }\end{array}$ & Tratamento endodôntico & $\begin{array}{l}\text { Sem informação para } \\
\text { RPTC } 1 \text { sessão } \\
31 \% \text { (RPTC } 2 \text { sessões) }\end{array}$ \\
\hline
\end{tabular}

Tabela 3. Tempo de acompanhamento e dados referentes às avaliações periódicas em cada estudo

\begin{tabular}{c|c|l|l}
\hline Tempo de acompanhamento & Número de avaliações & \multicolumn{1}{|c}{ Tipo de avaliações } & \multicolumn{1}{c}{ Autor } \\
\hline 6 meses & 2 & Clínica e Microbiológica & Petrou et al., 2014 \\
\hline 12 meses & 4 & Radiográfica e Clínica & Orhan et al., 2010 \\
\hline 12 meses & 1 & Radiográfica e Clínica & Bjørndal et al., 2010 \\
\hline 18 meses & 2 & Radiográfica & Oliveira et al., 2006 \\
\hline 18 meses & 1 & Radiográfica e Clínica & Maltz et al., 2013 \\
\hline 36 meses & $6-12$ & Radiográfica e Clínica & Mruythuysen et al., 2010 \\
\hline 36 meses & 1 & Radiográfica e Clínica & Leksell et al., 1996 \\
\hline 43 meses & 2 & Radiográfica e Clínica & Maltz et al., 2007 \\
\hline 45 meses & 3 & Radiográfica & Jardim et al., 2015 \\
\hline 72 meses & 4 & Radiográfica e Clínica &
\end{tabular}

\section{Discussão}

Estudos têm relatado que um tratamento conservador da cárie, associado a materiais antibacterianos e selamento adequado da cavidade, é capaz de promover a remineralização da dentina afetada, preservando a vitalidade pulpar e evitando exposição pulpar. ${ }^{4,9,11}$ Diferentes protocolos clínicos são sugeridos para a realização da RPTC, em substituição à RTTC em única sessão, sendo eles: tratamento em única sessão ${ }^{1,3,8,11,12}$ e/ou tratamento em duas sessões clínicas. ${ }^{3,4,6,8,9,11-13}$

Poucos estudos clínicos são encontrados na literatura avaliando a RPTC em dentes permanentes. Na presente pesquisa, foram encontrados apenas dez artigos que incluíram esse tipo de análise, apresentando as respectivas taxas de sucesso. Considerando até cinco anos de acompanhamento, aqueles que avaliaram a RPTC em sessão única, apresentaram uma taxa de sucesso variando de $78 \%$ a 99\% ${ }^{1,3,8,11,12}$ Já em relação à RPTC em duas sessões, a taxa de sucesso observada nos trabalhos selecionados variou de $53 \%$ a $94 \% .^{2,4,6,8,9,11-13}$

Após análise dos ensaios clínicos selecionados neste estudo, verificou-se que grande parte dos grupos tratados de maneira conservadora obteve índices de sucesso maiores do que aqueles tratados de forma mais radical, com remoção de todo o tecido cariado. Como exemplo, tem-se os estudos de Bjørndal et al. ${ }^{6} \mathrm{e}$ Orhan et al., ${ }^{11}$ que obtiveram taxas de sucesso na RPTC superiores (74,1\% e 94\%, respectivamente) às encontradas nos grupos tratados com RTTC (62,4\% e 75\%, respectivamente). 
Em relação aos trabalhos que compararam RPTC em sessão única e RPTC em duas sessões, ${ }^{3,8,11}$ as maiores taxas de sucesso foram encontradas quando se realizou a RPTC em uma única sessão (91\% a 99\%); na RPTC em duas sessões nesses trabalhos variou de $69 \%$ a $94,11 \%$.

Outro fator que influenciou diretamente o índice de sucesso foi o tempo de acompanhamento das restaurações. Isto foi bem observado no trabalho de Jardim et al., ${ }^{12}$ onde houve uma queda brusca nas taxas de sucesso dos dois tipos de RPTC's conforme aumentava o tempo de acompanhamento, chegando a apresentar $60 \%$ apenas de sucesso para RPTC 1 sessão versus $32 \%$ para RPTC 2 sessões, após 6 anos de acompanhamento. Para a RTTC, o tempo também influenciou negativamente, onde a taxa de sucesso chegou a ser $37,5 \%$ após 43 meses de acompanhamento. ${ }^{9}$

Dessa forma, quanto maior o tempo de duração dos controles, menores eram os índices de sucesso, provavelmente relacionados à maior susceptibilidade de fratura dentária, ${ }^{2,4}$ necrose pulpar, ${ }^{2,4}$ reabsorções ósseas apicaisl e perdas. ${ }^{2,4}$ Uma possível explicação para esse decréscimo, principalmente em decorrência de falhas relacionadas à vitalidade pulpar seria o diagnóstico incorreto da condição do tecido pulpar no momento da decisão do tratamento. ${ }^{4}$

É importante destacar as limitações existentes em trabalhos clínicos, uma vez que nesses estudos é necessária a colaboração do paciente no retorno para as avaliações periódicas. Alguns trabalhos tiveram uma perda de até $31 \%^{3,4,8}$ do tamanho inicial da amostra, o que pode ser considerado um viés de segmento e interferir no desfecho, aumentando os índices de sucesso.

Em um estudo retrospectivo avaliando somente a técnica da RPTC em sessão única, aplicando forramento com cimento de ionômero de vidro seguido de restauração permanente com resina composta, foi observada uma taxa de $93 \%$ de sucesso no tratamento de dentes permanentes após três anos de acompanhamento. ${ }^{1}$ Esses achados corroboram com a assertiva de que a dentina cariada residual (dentina desmineralizada) não representa uma ameaça à sobrevida da polpa, portanto não haveria a necessidade de expor o paciente a uma segunda escavação. ${ }^{5}$

Segundo Corralo e Maltz, ${ }^{7}$ os materiais mais comumente utilizados para induzir a remineralização da dentina cariada têm sido o cimento de hidróxido de cálcio (CHC) e o cimento de ionômero de vidro (CIV). O CHC em cavidades profundas é preferido por ser alcalino, induzir a remineralização e reduzir o risco de infecção bacteriana, além da biocompatibilidade. O CIV, por sua vez, possui adesividade à estrutura dentária, biocompatibilidade, libera flúor e também induz a remineralização, além de apresentar maior resistência mecânica que o $\mathrm{CHC}$, sendo ideal em cavidades médias.

Em relação à escolha dos materiais utilizados nas pesquisas avaliadas, houve uma variabilidade na sequência dos materiais, independente da técnica utilizada, mas basicamente os materiais de escolha tanto na RPTC quanto na escavação completa foram o CHC, ${ }^{3,4,6,6,10}$ IRM $^{3,4,8,10}$ e CIV, $, 1,3,6,8,10$ usados isoladamente ou associados. A resina composta foi o material definitivo de escolha em todos os artigos analisados.

$\mathrm{Na}$ RPTC duas sessões, a maioria dos autores elegeu para o desenvolvimento do tratamento na primeira sessão o forramento com hidróxido de cálcio pasta e restauração provisória com IRM. ${ }^{3,4,811}$ Depois de um determinado período, a restauração provisória era removida e substituída por CIV e restauração permanente normalmente com resina composta ${ }^{3,8,11}$ ou somente restauração permanente. ${ }^{2,4}$ No entanto, Bjørndal et al. ${ }^{6}$ utilizaram o CIV como material temporário na primeira sessão, enquanto que Orhan et al..$^{11}$ suprimiram o CIV tanto na primeira quanto na segunda sessão da RPTC.

Gruythuysen et al. ${ }^{1}$ foram os únicos autores que trabalharam com o CIV modificado por resina diretamente sobre a dentina profunda remanescente sem a aplicação prévia do $\mathrm{CaOH}$ e obtiveram taxas de sucesso relativamente satisfatórias (93\%). Dessa forma, é possível observar que o tratamento de cáries em cavidades profundas pela técnica da RPTC é bastante variável, não havendo, assim, um protocolo clínico definido quanto aos tipos de materiais e passos a serem realizados nessa modalidade de tratamento, dificultando ainda a comparação entre os trabalhos, uma vez que cada um segue um protocolo diferente.

É importante ressaltar que independente do material forrador utilizado na RPTC, o processo carioso estagna após a realização deste tratamento pulpar indireto, como foi mostrado no trabalho de Petrou et al., ${ }^{13}$ que utilizaram solução aquosa de hidróxido de cálcio, MTA e Portland Cement como forradores após RPTC 2 sessões e não obtiveram diferenças estatisticamente significantes entre os materiais e a taxa de sucesso foi alta para esse tipo de tratamento $(90,3 \%)$.

De acordo com Corralo e Maltz, ${ }^{7}$ não há diferenças de efeitos dos materiais de forramento CHC, IRM e CIV sobre a dentina cariada remanescente (dentina desmineralizada), sendo o sucesso do tratamento dependente de um bom diagnóstico da vitalidade do tecido pulpar e de um selamento adequado da cavidade, que vai impedir o crescimento bacteriano na camada da dentina afetada, estagnando, assim, o processo de cárie.

Por fim, as principais falhas observadas foram relacionadas à condição pulpar, onde a exposição pulpar foi a mais frequente..$^{2-4,6}$ Outras causas destacadas foram pulpite e necrose pulpar. De acordo com os resultados, as falhas foram mais evidentes durante a realização da RTTC, ${ }^{6,11}$ seguida pela técnica de RPTC em duas sessões, ${ }^{3,6,8,11}$ demonstrando, baseados nestes estudos clínicos, que a técnica da RPTC em sessão única apresenta melhor desempenho clínico.

\section{Conclusão}

Após a análise dos estudos clínicos incluídos nesta revisão integrativa, podem-se fazer as seguintes considerações finais:

- A RPTC, seja em sessão única ou duas sessões, parece ser uma alternativa viável para o tratamento de cáries profundas em dentes permanentes, visto que apresentou taxas de sucesso satisfatórias e superiores quando comparada à técnica da RTTC;

- A RPTC em sessão única apresentou melhores resultados do que a RPTC em duas sessões, sugerindo que não é necessária a reabertura da cavidade para remoção da dentina afetada remanescente;

- O hidróxido de cálcio pasta foi o material mais empregado tanto na técnica da RPTC em sessão única quanto em duas sessões, sugerindo que o presente material responde positivamente na reparação do remanescente dental cariado e preservação da vitalidade pulpar. No entanto, vale ressaltar que, independente do material utilizado, a técnica da RPTC bem executada responde positivamente em relação à estagnação do processo carioso. 


\section{Referências}

1. Gruythuysen R, Strijp GV, Wu MK. Long-term survival of indirect pulp treatment performed in primary and permanent teeth with clinically diagnosed deep carious lesions. JOE. 2010;36(9):1490-3.

2. Maltz M, Oliveira EF, Fontanella V, Carminatti G. Deep caries lesions after incomplete dentine caries removal: 40-month follow-up study. Caries Res. 2007;41:493-6.

3. Maltz M, Garcia R, Jardim JJ, de Paula LM, Yamaguti PM, Moura MS, et al. Randomized trial of partial vs. stepwise caries removal: 3-year follow-up. J Dent Res. 2012;91(11):1026-31.

4. Oliveira EF, Carminatti G, Fontanella V, Maltz M. The monitoring of deep caries lesions after incomplete dentine caries removal: results after 14-18 months. Clin Oral Invest. 2006;10:134-9.

5. Thompson V, Craig RG, Curro FA, Green WS. Treatment of deep carious lesions by complete excavation or partial removal: a critical review. J Am Dent Assoc. 2008;139(6):705-12.

6. Bjørndal L, Reit C, Bruun G, Markvart M, Kjaeldgaard M, Näsman P, et al. Treatment of deep caries lesions in adults: randomized clinical trials comparing stepwise vs. direct complete excavation, and direct pulp capping vs. partial pulpotomy. Eur J Oral Sci. 2010;118(3):290-7.

7. Corralo DJ, Maltz M. Clinical and ultrastructural effects of different liners/res- torative materials on deep carious dentin: a randomized clinical trial. Caries Res. 2013;47(3):243-50.

8. Maltz M, Jardim JJ, Mestrinho HD, Yamaguti PM, Podestá K, Moura MS, et al. Partial removal of carious dentine: a multicenter randomized controlled trial and 18-month follow-up results. Caries Res. 2013;47(2):103-9.

9. Leksell E, Ridell k, Cvek M, Mejàre I. Pulp exposure after stepwise versus direct complete excavation of deep carious lesions in young posterior permanent teeth. Endod Dent Traumatol. 1996;12(4):192-6.

10. Schwendicke F, Meyer-Lueckel H, Dörfer C, Paris S. Failure of incompletely excavated teeth-a systematic review. J Dent. 2013;41(7):569-80.

11. Orhan AI, Oz FT, Orhan K. Pulp exposure occurrence and outcomes after 1- or 2 -visit Indirect pulp therapy vs complete caries removal in primary and permanent molars. Pediatric Dent. 2010;32(4):347-55.

12. Jardim JJ, Simoneti MND, Maltz M. Remoção parcial de tecido cariado em dentes permanentes: seis anos de acompanhamento. RFO, Passo Fundo. 2015;20(1):39-45.

13. Petrou MA, Alhamoui FA, Welk A, Altarabulsi MB, Alkilzy M, Splieth CH. A randomized clinical trial on the use of medical Portland cement, MTA and calcium hydroxide in indirect pulp treatment. Clin Oral Invest. 2014;18(5):1383-9.

\section{Mini Currículo e Contribuição dos Autores}

1. Joyce Figueira de Araújo - cirurgiã-dentista e mestre em Odontologia. Contribuição: concepção, preparação e redação do manuscrito.

2. Érica Martins Valois - cirurgiã-dentista e mestre em Odontologia. Contribuição: preparação e redação do manuscrito.

3. Andréa Dias Neves Lago - cirurgiã-dentista e doutora em Dentística. Contribuição: levantamento dos artigos para a revisão integrativa.

4. Breno Mont Alverne H. da Silva - cirurgião-dentista e doutor em Odontologia. Contribuição: delineamento do estudo e levantamento dos artigos.

5. José Ferreira Costa - cirurgião-dentista e doutor em Odontologia. Contribuição: revisão crítica e aprovação final.

6.Leily Macedo Firoozmand - cirurgiã-dentista e doutora em Odontologia. Contribuição: delineamento do estudo, revisão crítica e aprovação final.

Recebido em: 20/11/2016 / Aprovado em: 02/01/2017

Autor Correspondente

Joyce Figueira de Araújo

E-mail: joycefa1@hotmail.com 